\title{
Removal of Etalon Features in the Far-infrared/THz Transmittance Spectra of Thin Polymer Films
}

\author{
Ulrich Schade ${ }^{1}$, Dawei Cao ${ }^{1}$, Ljiljana Puskar ${ }^{1}$, Eglof Ritter ${ }^{1}$ and Jörg Beckmann ${ }^{2}$ \\ ${ }^{1}$ Helmholtz-Zentrum Berlin für Materialien und Energie, Albert-Einstein-Str. 15, 12498 Berlin, \\ Germany \\ ${ }^{2}$ Bundesanstalt für Materialforschung und -prüfung, Unter den Eichen 87, 12489 Berlin, Germany
}

\section{Corresponding author:}

Ulrich Schade,

E-mail: Ulrich.Schade@Helmholtz-Berlin.de

\begin{abstract}
Etalon features in infrared spectra of stratified samples, their influence on the interpretation and methods to circumvent their presence in infrared spectra have been in discussion for decades. This paper focuses on the application of a method originally developed to remove interference fringes in the mid-infrared spectra for far-infrared Fourier Transform spectroscopy on thin polymer films. We show that the total transmittance-reflectance technique, commonly used for mid-infrared, also works successfully in the far infrared spectral range where other approaches fail. Experimental spectra obtained by such technique are supported by model calculations and reveal the possibility and limits to obtain almost undisturbed far-infrared spectra which are suitable to determine low energy vibrations of ionomer salts under certain sample conditions.
\end{abstract}

\section{Keywords}

Far infrared spectroscopy, absorption, etalon feature, total transmittance reflectance, wedged film

\section{Introduction}

Far-infrared/THz spectroscopy is a widely used tool to study the molecular dynamics and the intermolecular interactions in polymers from which chemical and physical properties can be deduced. Of special interest are far-infrared studies of proton exchange membranes (PEMs) as they play the crucial role in the operation of fuel cells for clean energy conversion. Essential parameters of 
such membranes in order to ensure a good fuel cell performance are high proton conductivity while maintaining adequate mechanical-chemical stability. For Nafion ${ }^{1}$, a widely used ionomer based on polytetrafluoroethylene (PTFE) fulfils this prerequisites at wet conditions and operating temperatures below $80^{\circ} \mathrm{C}$. The technological quest for novel high performance PEMs which operate at higher temperatures and drier conditions is directed towards new multi-acid side-chain ionomers. ${ }^{2-5}$ Here, essential information on the functionality of such membranes can be obtained by infrared spectroscopic methods.

As an example, the acid sites of the side chains can be probed by measuring the far-infrared absorption of cation motion of the PEM salt form in thin film samples. The effective force constant for the forces between the ionomer anion site and the counter cations can easily be obtained from the vibrational frequencies between 1 and $10 \mathrm{THz}\left(30-300 \mathrm{~cm}^{-1}\right) \cdot{ }^{6-8}$ The knowledge of the vibrational frequencies of cations with different atomic weight and oxidation state may support further development of more accurate force field models as well as more accurate molecular dynamics simulations and quantum mechanical calculations. ${ }^{9-12}$ Transmittance measurements of free-standing PEM materials seem here to be the simplest experimental approach in order to obtain quantitative far-infrared absorbance data. However, experimental conditions exist, where etalon features (interference fringes) mimic the existence of additional bands and disturb or even hinder the quantitative interpretation of absorption spectra of thin ionomer membranes ${ }^{8}$ with technologically relevant thicknesses below $100 \mu \mathrm{m}$.

This paper reports on the far-infrared/THz application of the total transmittance reflection (TTR) techniques for the analysis of thin polymer films. The technique is exemplarily employed on thin Nafion films in order to uncover the undisturbed absorption bands of the cation vibrations. The TTR technique in the far infrared may pave the way to other applications such as the investigation of the microstructure and the morphology of polymer films with respect to orientation states, crystallinity and interphase content. The range of application of the technique is derived by means of model calculations and proved by far-infrared Fourier Transform (FT) spectroscopy measurements on PTFE films of different thicknesses.

\section{Strategies for the removal of etalon features}

Etalon features are the result of a coherent adding up of waves caused by multiple reflections at the internal boundaries where the optical constants abruptly change. Such boundaries exist for a freestanding polymer film in contact with the ambient (air), where the optical constant changes from the value typical for the polymer material to unity. Multiply reflected waves from the film boundaries reach the detector position where they experience a constructive or destructive superposition 
according to the apparent phase shift. This in turn produces a sinusoidal oscillation in the absorption spectra and may influence the measured absorption band positions, dependent on the thickness and dielectric properties of the film. ${ }^{13,14}$

Several more or less effective technical, mathematical and optical methods exist to minimize this effect in order to get meaningful and evaluable spectra. Such methods are primarily discussed for the mid-infrared spectral range ${ }^{15-24}$ and will be reviewed and checked for applicability in the far infrared in this paragraph with no claim for completeness.

A simple way to depress interference fringes in the transmittance spectra is achieved by placing the film onto a suitable substrate to artificially thicken the film. The intimate contact between the film and the substrate provides no abrupt change of the refractive index at the interface and the achieved overall thickness pushes the fringe spacing below the spectral resolution of the spectrometer. ${ }^{25}$ However, the substrate should be spectrally transparent and featureless in the region investigated to avoid interferences with the spectral information of the sample itself.

A mathematical treatment for fringe removal in films for transmittance spectra is given by Neri et $a l^{17}{ }^{17}$. The treatment can directly be performed within most current commercial spectrometer software. However, the method is restricted to equidistant fringes and to absorption band widths larger than the fringe distance itself. While this method may work quite well in the mid-infrared for film thicknesses in the tens-micrometer range, it seems to be unsuitable for the far-infrared range, where the distances of adjacent fringes are similar to the widths of the absorption bands, which might be distorted or even be removed by this treatment as well.

A partial destruction of the coherence to hinder the occurrence of fringes can be provoked by roughening the surface of the film. Again, this method seems appropriate only for the midinfrared spectral range. To avoid fringes in the far-infrared spectra of thin films, a roughness of the order of the actual film thickness would be necessary which is physically impossible to establish. $<$ Insert Figure 1 here $>$

Another spectroscopic strategy to "destroy" coherence is based on wedged film boundaries. This is successfully used in the mid-infrared spectral range for the construction of cuvettes and optical windows. ${ }^{21,22,26}$ Here, opposite optical boundaries are not anymore parallel which prevents a fully coherent superposition of waves and therefore suppresses the appearance of fringes. However, in thin films there is a physical limit for sufficiently large wedge angles. As shown in Fig. 1 the fringe amplitudes become strongest in the far-infrared spectral range and only slowly decrease with increasing wavenumbers, again favoring the method for the acquisition of thin film spectra in the mid-infrared. 
Harrick $^{27}$ proposed a method for fringe free transmittance spectra by striking the film with $p$ polarized light at the Brewster angle of the film material. The reflectivity for both interfaces becomes zero and hinders the multiple reflections in the film and thereby avoids the oscillation in the spectra. $<$ Insert Figure 2 here>

Another optical method to cancel oscillations was proposed by Edgar and Stay ${ }^{23}$ using a plane mirror situated behind and adjacent to the film. The light passing through the film is reflected off the mirror to pass through the film from the back a second time so that the final absorption is doubled. This so-called mirror backed reflection or total transmittance reflection (TTR) technique has been successfully employed in the mid-infrared spectral range in the analysis of free-standing thin films and thin films on transparent substrates. ${ }^{18-20} \mathrm{~A}$ scheme of the TTR technique is presented in Fig. 2. It shows that the detected intensity is built up from the coherent superposition of all reflected and transmitted waves. The depression of interference fringes is assumed by the fact that the destructive interference of the waves transmitting the film should be balanced by the constructive interference of those reflected waves experiencing a $180^{\circ}$-phase change upon reflection. However, sufficiently complete depression of fringes is only possible if the overall reflection of the film-mirror set-up is not disturbed too much by the optical film properties and is close to $100 \%{ }^{19}$, i.e., a weak film absorption and weak reflection at the film boundaries are requested. It should be mentioned, that a quite common transflectance technique in IR microscopy ${ }^{28}$ differs from the above mentioned method by former lacking an air gap between the film and the back-reflecting medium, which tends to produce interference fringes in the spectra.

\section{Experimental Procedures and Modelling}

Nafion films with a nominal thickness of $25.4 \mu \mathrm{m}$ and $50.8 \mu \mathrm{m}$ and an equivalent weight (EW) of

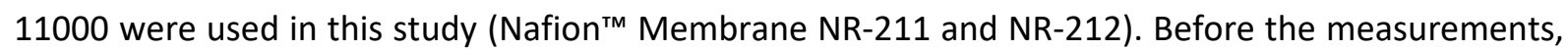
the Nafion films underwent a standard pre-treatment procedure ${ }^{29}$ consisting of boiling steps at $80{ }^{\circ} \mathrm{C}$ in $3 \% \mathrm{H}_{2} \mathrm{O}_{2}$, deionized (DI) water and in $0.5 \mathrm{M} \mathrm{H}_{2} \mathrm{SO}_{4}$ to fully convert Nafion into the acid form. The salt forms of the Nafion films were obtained by soaking in $1 \mathrm{M}$ high purity alkali salt ( $\mathrm{LiCl} 99 \%, \mathrm{NaCl}$ 99.5\%, $\mathrm{KCl} 99 \%, \mathrm{RbCl} 99 \%$ and $\mathrm{CsCl} 99.999 \%)$ solutions for about 12 hours. Subsequent to the soaking, the films were rinsed in deionized water for few seconds and dried in ambient air between lens paper sheets. Before the spectral investigation an additional drying was conducted in vacuum employing the environment of the spectrometer sample compartment $\left(<1 \mathrm{mbar}, 26^{\circ} \mathrm{C}\right)$. Drying was considered to be completed in less than one hour when no further change in water content could be observed in the mid-infrared spectrum of the Nafion film. 
In addition, PTFE films with thicknesses between $10 \mu \mathrm{m}$ and $200 \mu \mathrm{m}$ were investigated. These films can serve as an optical model for Nafion, because the $\left(C_{2} F_{4}\right)_{n}$ main chain of PTFE represents also the backbone of Nafion. The PTFE films were rinsed in acetone and 2-propanol prior to the measurements in order to remove possible organic contaminations. It should be mentioned, that the crystallinity of the polymers and the drawing direction of the different films are not specifically considered for a later spectral evaluation.

The samples were measured in raw vacuum $(<1 \mathrm{mbar})$ at room temperature in the spectrometer compartment in normal-incidence and in transmittance geometry either by using a Bruker $66 / v$ or a Bruker $80 / v$ FT-IR spectrometer with $1 \mathrm{~cm}^{-1}$ spectral resolution. The Bruker 66/v was operated with a broadband Mylar beamsplitter and the Bruker 80/v spectrometer with a Si beamsplitter. An interferometer scan velocity of $1.27 \mathrm{~cm} / \mathrm{s}$ was chosen for both spectrometers. A number of interferometer scans were co-added in order to improve the signal-to-noise ratio (SNR). The appropriate numbers of scans were adopted according to the different experimental set-ups used to achieve a reasonable signal-to-noise (SNR) value. A mercury-arc lamp and, when necessary, synchrotron radiation as infrared source were used. The mercury-arc lamp required the accumulation of scans over a longer period of time to achieve a sufficient SNR down to about $50 \mathrm{~cm}^{-1}$. However, spectra with good SNR down to $15 \mathrm{~cm}^{-1}$ could be achieved with the synchrotron source in a much shorter period of time. The spectral region between 25 and $5 \mathrm{~cm}^{-1}$ was covered by infrared synchrotron radiation having the electron storage ring operating in a so-called "low a" mode resulting in a coherent enhancement of several orders of magnitude in intensity over the mentioned spectral range. ${ }^{30}$ Spectra were detected with a 4.2-K Si composite bolometer using entrance filters of 100 and $600 \mathrm{~cm}^{-1}$. In order not to overload the bolometer when coherent synchrotron radiation was used, an additional quartz filter was applied as an attenuator. Spectra of the different spectral ranges obtained by the different detector filters and radiation sources were merged in order to display the complete spectral range.

The quasi normal incidence set-up used in this study enables measurements of the single channel reflectance of a sample with a fixed angle of incidence/emergence of $8^{\circ}$. In order to perform TTR measurements an aluminum mirror was placed at the sample position and the film under investigation is situated in the beam path in an adjustable distance from this mirror. The film itself is clamped in a frame to ensure certain flatness. The mirror and the film are adjusted parallel to each other before the measurement by means of a kinematic mount which is controlled via an autocollimation procedure using a visible laser. Two flipping mirrors allow for the beam passing either through the set-up or through the empty sample compartment of the spectrometer. The latter is used as an internal reference for the measurement of either (1) the sample mirror combination or (2) the sole aluminum mirror. These two arrangements can be measured independently without losing 
the relation to each other. These reference measurements account for any possible slow drifts in the spectral content of the radiation source over a longer time of data acquisition. In addition, each arrangement can be measured multiple times and averaged for a statistical improvement. Finally, the TTR spectra are obtained by rationing the averaged sample measurements and the averaged measurements of the aluminum mirror.

The transmittance measurements were performed by employing an 8-position sample wheel in the focus position of the sample compartment and using an empty position as reference. The circular sample size has a diameter of $13 \mathrm{~mm}$ for the sample wheel and $25 \mathrm{~mm}$ for the TTR set-up ensuring an almost diffraction-free investigation of the polymer films down to $5 \mathrm{~cm}^{-1}$. Both set-ups are controlled by a macro language offered by the spectrometer software. The experimental absorbance spectra were calculated as the negative logarithm of the transmittance and TTR spectra, respectively. Since all the measurements were performed in normal or quasi-normal incidence the influence of partial polarization of the infrared beam to the experimental results could be neglected.

In order to model TTR absorbance spectra the film-mirror system is treated as an isotropic stratified structure with three interfaces such as (i) ambient-first film boundary, (ii) second film boundary-ambient and (iii) ambient-mirror. Analytical approaches for such multi-layer system ${ }^{31-33}$ provide the complex amplitude reflectance, $r_{s, p}(\nu, \alpha)$, which describes the change in amplitude and phase of a planar electromagnetic wave upon specular reflection for an angle of incidence, $\alpha$, for the field components perpendicular ( $s-)$ and parallel ( $p-$ ) to the plane of incidence. The TTR spectrum, $S_{T T R}$, for non-polarized radiation as obtained by conventional far-infrared dispersive or FT spectroscopy is then given by the square of the magnitudes of the complex amplitude reflectance, $r_{s, p}(v)$, and reads:

$$
S_{\text {TTR }}=\frac{\left|r_{p}(v, \alpha)\right|^{2}+\left|r_{s}(v, \alpha)\right|^{2}}{2} .
$$

The TTR spectrum is converted into absorbance by:

$$
A_{T T R}=-\log \left(S_{T T R}\right) \text {. }
$$

The transmittance spectra, $S_{T}$, is calculated from the transmitted field components $t_{p}$ and $t_{s}$ in the same way by treating the film as an isotropic stratified structure with two interfaces, (i) the ambientfirst film boundary and (ii) the second film boundary-ambient, respectively, and is given by: 
$S_{T}=\frac{\left|t_{p}(v, \alpha)\right|^{2}+\left|t_{s}(v, \alpha)\right|^{2}}{2}$,

which can be converted into an absorbance spectrum according to

$A_{T}=-\log \left(S_{T}\right)$.

Generally, the absorbance is taken as a measure of the extinction caused by the related species present in the sample and is expressed by the Lambert-Beer law:

$A_{L B}=4 \pi v k x$

where $v$ is the frequency in wavenumbers, $k$ is the extinction coefficient and $x$ is the sample thickness.

\section{Results and Discussion}

For the case of the free-standing films discussed in this paper, the flux passing through the films in not only attenuated by the absorption of the film material according to the Lambert-Beer law but is also attenuated by reflection at the first film boundary and by further multiple internal reflections initiated at the second film boundary. ${ }^{34}$ This causes an erroneous absorbance value, especially when weak absorbers or almost transparent films are involved. Therefore, it is of certain importance for quantitative statements how much and under which experimental conditions (e.g., thickness and oscillator strength) the absorption values obtained from the TTR and transmittance geometry deviate from the Lambert-Beer law.

To address this, PTFE films with thicknesses varying from 10 to $200 \mu \mathrm{m}$ were measured between 50 and $300 \mathrm{~cm}^{-1}$. The obtained absorbance spectra in both transmittance and total transmittance reflection geometry are shown in Fig. 3. The spectra are generally governed by the strong backbone absorption at $203 \mathrm{~cm}^{-1}$ caused by the rock/torsion vibration of neighboring $\mathrm{CF}_{2}$ groups. ${ }^{35}$ Almost no absorption in PTFE occurs in the spectral region below that band, except for some very weak lattice modes ${ }^{36}$ which are not resolved in any of the samples used in this study. For wavenumbers larger than $203 \mathrm{~cm}^{-1}$ some vibrational bands appear (e.g., at $277 \mathrm{~cm}^{-1}$ and $293 \mathrm{~cm}^{-1}$ which can be assigned to $\mathrm{CF}_{2}$ wagging ${ }^{37}$ and twisting vibrations ${ }^{38}$, respectively). Typical interference fringes arise in the "absorption-free" region of the transmittance spectra. The period of these fringes decreases with increasing film thickness while the fringe amplitude is independent of the film 
thickness within the accuracy of the measurements. The fringes are successfully depressed in the corresponding experimental TTR spectra for film thicknesses up to $100 \mu \mathrm{m}$. In addition, the absorbance values become very low in this spectral range, except for the thickest film measured. The spectra for both the transmittance and TTR method develop differently in the absorption regions if the film thickness increases. While for the transmittance spectra the absorbance band increases with increasing film thickness as one would expect, it appears that for the TTR spectra the absorbance band at $203 \mathrm{~cm}^{-1}$ starts to saturate, changes the shape and even shifts towards higher wavenumbers.

$<$ Insert Figure 3 here $>$

$<$ Insert Figure 4 here>

For comparison we performed model calculations only considering the strong backbone vibration in PTFE. This vibration band can be described by a simple Drude-Lorentz oscillator model ${ }^{39}$ rendering the frequency dependence of the permittivity, $\varepsilon$, or rather optical constants, $\tilde{n}$, of the film material:

$$
\begin{aligned}
& \varepsilon^{\prime}=\varepsilon_{\infty}+\frac{F\left(v_{0}^{2}-v^{2}\right)}{\left(v_{0}^{2}-v^{2}\right)^{2}+(\Gamma v)^{2}} \\
& \varepsilon^{\prime \prime}=\frac{F(\Gamma v)}{\left(v_{0}^{2}-v^{2}\right)^{2}+(\Gamma v)^{2}} \\
& \tilde{n}=n+i k=\sqrt{\varepsilon^{\prime}+i \varepsilon^{\prime \prime}},
\end{aligned}
$$

applying an oscillator frequency $v_{0}=200 \mathrm{~cm}^{-1}$, an oscillator strength $F=10^{3} \mathrm{~cm}^{2}$, a damping $\Gamma=10 \mathrm{~cm}^{-1}$ and an $\varepsilon_{\infty}=2$. An realistic optical constant for aluminum in the far infrared of $(100+i 200)^{40}$ is used for the back-reflecting metal mirror, which is $5 \mathrm{~mm}$ off the film.

The calculated absorbance spectra for different film thicknesses calculated for a spectral resolution of $2 \mathrm{~cm}^{-1}$ for the transmittance and TTR geometry are shown in Fig 4. The dominant spectral feature of the measurements shown in the Fig. 3 is well retrieved by the calculated spectra. The shift of the absorption band towards higher wavenumbers and the saturation behavior observed in the experimental TTR spectra are replicated in the theoretical TTR spectra. With increasing of the film thickness the band distortion develops towards a dispersion-like feature calculated for the reflection of an infinitely thick film. The fringes in spectral regions of no absorption which are clearly 
visible in the transmittance geometry are depressed in the TTR geometry with the TTR absorbance in the same regions reaching a value close to zero.

$<$ Insert Figure 5 here>

In Fig. 5 it is shown how the absorbance maxima for the bands at 203 and $277 \mathrm{~cm}^{-1}$ in the experimental spectra of single transmittance and TTR geometry develop with film thicknesses. These band maxima are also compared to a straight line fit assuming a Lambert-Beer behavior. The transmittance absorbance maximum at each point (thickness) for both bands follows the fit. On the other hand, the TTR absorbance band maximum becomes remarkably nonlinear for film thicknesses above $100 \mu \mathrm{m}$ for the $277-\mathrm{cm}^{-1}$ band and for the case of $203-\mathrm{cm}^{-1}$ band already starts to saturate for film thicknesses above $50 \mu \mathrm{m}$. The zero thickness extrapolated values of the absorbance maxima obtained in transmittance geometry are close to the value one would expect from the reflection losses originated solely at the film boundaries (e.g., of 0.026 a.u. for $n=1.41$ ). In contrast, the extrapolation of the TTR maxima to zero film thickness does not indicate such extenuation and gets close to zero.

$<$ Insert Figure 6 here>

Finally, Nafion films, which had been soaked in several alkali salt solutions and subsequently dried prior to the spectral investigation, were measured both in total transmittance reflection and transmittance geometry. The films were thin enough not to be strongly affected by the distortion discussed above for thicker PTFE films. In Fig. 6 the TTR absorption spectra of a Nafion $/ \mathrm{K}^{+}$film of a thickness of $25.4 \mu \mathrm{m}$ are shown as a function of the gap between the rear side of the film and the back-reflecting mirror. As the length of the gap gets shorter the spectra become more and more disturbed by interference fringes of decreasing fringe distance and increasing modulation strength. These fringes are caused by multiple reflections formed in the gap between the interfaces. The fringes abruptly disappear in the spectrum when the film touches the mirror. For this case, the set-up turns into the aforementioned transflection geometry where the interpretation of the spectrum is severely distorted by internal reflection in the film depending on film thickness itself and the dielectric properties of the film and back material. ${ }^{28}$ In contrast, when a sufficiently thick gap is chosen the interference fringes of the gap are not anymore resolvable and are averaged out by the spectral resolution of the instrument. This happens in our case at a distance of $5.5 \mathrm{~mm}$ for a spectral resolution of $1 \mathrm{~cm}^{-1}$ and makes an interpretation of the absorption bands likely. Now clearly seen in the spectra is the cation vibration of potassium ${ }^{7}$ at around $150 \mathrm{~cm}^{-1}$ and other bands, which can be related to the PTFE backbone, for example the characteristic band at about $203 \mathrm{~cm}^{-1}$ and some weaker bands at higher frequencies, (e.g., at $277 \mathrm{~cm}^{-1}$ and $293 \mathrm{~cm}^{-1}$ as seen in the PTFE spectrum). $<$ Insert Figure 7 here> $<$ Insert Figure 8 here> 
Fig. 7 and Fig. 8 compare the TTR with the transmittance absorbance spectra of a Nafion $\mathrm{NR} 211 / \mathrm{K}^{+}$film of a thickness of $25.4 \mu \mathrm{m}$ and a Nafion NR212/Cs ${ }^{+}$film of a thickness of $50.8 \mu \mathrm{m}$, respectively. As expected the absorbance obtained from the TTR geometry approximately doubles the absorbance obtained from the transmittance measurement since the radiation passes the film under investigation twice. A closer inspection of the spectra reveals remarkable differences when comparing the two measurement modes. The position of the $\mathrm{K}^{+}$absorption shifts from $154 \mathrm{~cm}^{-1}$ in the transmittance spectrum to $150 \mathrm{~cm}^{-1}$ in the TTR spectrum and the position of the $\mathrm{Cs}^{+}$absorption from $92 \mathrm{~cm}^{-1}$ in the transmittance spectrum to $83 \mathrm{~cm}^{-1}$ in the TTR spectrum. Furthermore, the relative strength of the cation band in relation to the backbone absorption band at $203 \mathrm{~cm}^{-1}$ is different and especially noticeable for the Nafion NR211/ $\mathrm{K}^{+}$. In addition, the transmittance spectra show bands which cannot be found in the TTR spectrum. This is particularly obvious for spectral features at 33 and $162 \mathrm{~cm}^{-1}$ in the transmittance absorbance spectrum of Nafion/Cs ${ }^{+}$and at about $70 \mathrm{~cm}^{-1}$ in the transmittance absorbance spectrum of Nafion/ $\mathrm{K}^{+}$. Finally, bands beyond $200 \mathrm{~cm}^{-1}$ are completely different in their shape if compared to their related TTR absorbance spectrum. All these spectral changes in the transmittance absorbance spectra are in fact distortions caused by multiple reflections within the film and may lead to qualitative misinterpretations and quantitative errors of derived band parameters.

In an earlier study on alkali salts of the two ionomers Nafion and perfluoroimide $\operatorname{acid}^{8}$ a simple model was applied to account for the influence of film interferences when deriving absorption band parameters from film measurements. The calculated absorbance spectrum of a non-absorbing film of same thickness (and refractive index) of the sample film ${ }^{13}$ was subtracted from the measured absorbance spectrum which yielded in an almost undisturbed film spectrum and allowed deriving the band positions of the alkali ion vibrations. Having now measured the sample in TTR and transmittance geometry this simple approach can easily be evaluated. Fig. 7 and 8 also display the difference between the transmittance absorbance and the TTR absorbance spectra for Nafion NR211/K $\mathrm{K}^{+}$and Nafion NR212/Cs ${ }^{+}$. Before the subtraction the TTR absorbance spectra had been divided by two in order to account for the double beam path through the film. The differences of the experimental spectra show a typical fringe pattern and were compared to the absorbance spectra of modelled non-absorbing films for the two thicknesses of 26 and $52 \mu \mathrm{m}$, respectively. The model spectra were calculated by applying the refractive index of PTFE of 1.41 for very low wavenumbers outside of the $203-\mathrm{cm}^{-1}$ absorption region. ${ }^{41}$ The spectra of the modelled films describe the experimental difference spectra reasonably well. However, there is a larger discrepancy between the non-absorbing model and the experiment in spectral regions, where either stronger background 
absorption and/or distinct absorption bands exist. In these regions the refractive index of the Nafion films differs significantly from that of the model film since the refractive index is coupled to the extinction coefficient via the Kramers-Kronig dispersion relation.

$<$ Insert Table 1 here $>$

Finally, Table 1 displays the band position derived from TTR absorbance spectra for the potassium and cesium salts of Nafion in comparison with data obtained through the model above and with older transmittance data of Matera et al. ${ }^{7}$, to our knowledge the only available data from literature. Unfortunately, there is no information given on the experimental conditions the data of Matera et al. ${ }^{7}$ are taken, except that the spectral resolution of the spectra presented is $4 \mathrm{~cm}^{-1}$. So, it is dubious to compare it with the other data of Table 1, each derived from films of comparable thickness. However, here both data for the band position of potassium and cesium salts are different. The differences cannot be explained with the optical resolution that is $1 \mathrm{~cm}^{-1}$ in each case. Unless other experimental influences have to be considered, the differences can be sure indicate that the subtraction method ${ }^{8}$ is less accurate than the TTR approach. Since only few data are available to prove, further experiments on Nafion salts have to be made for clarification.

\section{Summary}

Several strategies to minimize or even eliminate etalon effects in infrared spectra are discussed in terms of their applicability to the far infrared spectral region. Most of them fail in that region due to the unfavorable sample geometry at longer wavelengths or due to the similar width of absorption bands and fringes. Finally, the subtraction of a calculated spectrum of a non-absorbing film from the experimentally determined spectrum becomes incorrect in spectral regions of stronger absorption.

Here we show that the total transmittance reflection (TTR) technique successfully removes the interference fringes in FT-IR spectra of thin polymer films in the far-infrared/THz. In addition, losses due to the reflection at the film boundaries are almost eliminated, which is of importance for transmission measurements of weakly absorbing films when deriving precise extinction coefficients. The method works well when the gap distance between film and reflecting mirror is chosen such that the related interference fringes from the gap are averaged out by the spectral resolution given by the spectrometer. However, for thicker films and/or for stronger absorption, a distortion of the absorption band can become apparent and can finally reach the shape of a feature typical for reflectance spectra from bulk material.

As exemplarily shown for a few alkali salt samples of Nafion, the TTR technique allows determining low energy vibrational energies in ionomer films in the far-infrared/THz spectral range. 


\section{Appendix: The Transmittance Spectrum of a wedged Film}

A simple analytical expression for the transmittance of a wedged film, as it becomes necessary for the discussion of sample geometries for simple fringe suppression in the far infrared spectral range, could not be found in the literature and is therefore derived below.

Let us consider a non-absorbing film with parallel boundaries, a refractive index of $n$, a thickness of $x$ and vacuum as ambient. The transmitted light passing in direction normal to the film contains a superposition of electromagnetic waves multiply reflected inside the film at both the film boundaries. These waves undergo a phase change, $\beta$, each time they travel from one boundary to the other

$\beta=4 \pi v n x$,

Summing up the transmitted partial wave amplitudes yields the amplitude transmittance

$t=\frac{t_{1} t_{2} e^{i \frac{\beta}{2}}}{1+r_{1} r_{2} e^{i \beta}}$

where $t_{1,2}$ and $r_{1,2}$ are the corresponding Fresnel coefficients of the first and second film boundary, respectively, and the following applies: $r_{1}=-r_{2}, R=r_{1} r_{2}, T=t_{1} t_{2}$ and $1=T+R$. The well-known Airy formula for the transmittance of a non-absorbing film is then given by

$$
A(v)=t t^{*}=\frac{T^{2}}{1-R^{2}} \frac{1-R^{2}}{1+R^{2}-2 R \cos \beta},
$$

which, for instance, can be averaged numerically along the edge direction ${ }^{15}$. For an analytical approach we consider that the wedged film linearly varies its thickness over the illuminated area from $x_{1}$ to $x_{2}$ and the infinitesimal averaging of the Airy formula reads:

$$
T_{\text {wedge }}(v)=\frac{1}{\left|x_{1}-x_{2}\right|} \int_{x_{1}}^{x_{2}} A(v) d x
$$

For the analytical solution of the Airy formula it is of help to transform the following part of the integrant into a trigonometric series: ${ }^{42}$ 


$$
\frac{1-R^{2}}{1+R^{2}-2 R \cos \beta}=1+2 \sum_{k=1}^{\infty} R^{k} \cos k \beta
$$

The integration followed by rearranging and simplifying gives the transmittance of the wedge, where $x_{d}=\left|x_{1}-x_{2}\right|$ stands for the difference between the thickest and the thinnest part of the illuminated part of the wedge and $x_{m}=\left(x_{1}+x_{2}\right) / 2$ for the corresponding average thickness:

$$
T_{\text {wedge }}(v)=\frac{1-R}{1+R}\left(1+\sum_{k=1}^{\infty} \frac{R^{k}}{\pi k n v x_{d}} \sin \left(2 \pi k n v x_{d}\right) \cos \left(4 \pi k n v x_{m}\right)\right) .
$$

For very small wedge angles, Eq. A6 evolves into

$$
\lim _{x_{d} \rightarrow 0} T_{\text {wedge }}(v)=\frac{1-R}{1+R}\left(1+2 \sum_{k=1}^{\infty} R^{k} \cos (4 \pi k n v x)\right) \text {, }
$$

which can be written back into the known form of the Airy formula (Eq. A3) applying the same trigonometric series as used for Eq. A5.

Eq. A6 is composed of a term which describes the transmittance of a film with parallel boundaries, where the multiple reflected waves are incoherently treated, i.e., their intensities are summed up. To this term a series is added which counts for the fringes by two beating sin- and cosfunctions. The series in Eq. A6 converges rather fast since $R<1$. It is already sufficiently precise to consider in Eq. A6 the first or the first and the second term of the series depending on the value of the refractive index of the wedge material. The amplitude of the fringes decreases for an increase of the difference between the thickest and the thinnest part of the illuminated wedge, $x_{d}$, and generally falls off by $1 / v$ with increasing wavenumber.

\section{Acknowledgement}

U. S. acknowledges fruitful discussions with A. Röseler. E. R. acknowledges support from the German Federal Ministry of Education (BMBF) Research Grant 05K13KH1. D.C. acknowledges support from the China Scholarship Council (CSC).

\section{Conflict of Interest}


The authors declare no conflict of interest.

\section{References}

1. A.K. Sahu, S. Pitchumani, P. Sridhar, A.K. Shukla. "Nafion and modified-Nafion membranes for polymer electrolyte fuel cells: An overview". Bull. Mater. Sci. 2009. 32(3): 285-294. 10.1007/s12034-009-0042-8.

2. M. Schaberg, J. Abulu, G. Haugen, M. Emery, S. O'Conner, P. Xiong, S. Hamrock. "New multi acid side-chain lonomers for proton exchange membrane fuel cells". ECS Trans. 2010. 33(1): 627-633. 10.1149/1.3484559.

3. M. Danilczuk, L. Lancucki, S. Schlick, S.J. Hamrock, G.M. Haugen. "In-Depth Profiling of Degradation Processes in a Fuel Cell: 2D Spectral-Spatial FTIR Spectra of Nafion Membranes". ACS Macro Lett. 2012. 1(2): 280-285. 10.1021/mz200100s.

4. C. Marcott, T. Awatani, J. Ye, D. Gerrard, M. Lo, K. Kjoller. "Review of nanoscale infrared spectroscopy applications to energy related materials". Spectrosc. Eur. 2014. 26(1): 18-22.

5. L. Puskar, E. Ritter, U. Schade, M. Yandrasits, S.J. Hamrock, M. Schaberg, E.F. Aziz. "Infrared dynamics study of thermally treated perfluoroimide acid proton exchange membranes". Phys. Chem. Chem. Phys. 2017. 19(1): 626-635. 10.1039/C6CP06627E.

6. V.D. Mattera, S.L. Peluso, A.T. Tsatsas, W.M. Risen. "Spectroscopic and thermal studies of ionic interactions in ionomers". ACS Symp. Ser. 1986. 320: 54-65.

7. V.D. Mattera, W.M. Risen. "A far-infrared study of ionic interactions in poly(styrene sulfonic acid) ionomers". J. Polym. Sci. Polym. Phys. Ed. 1984. 22(1): 67-77. 10.1002/pol.1984.180220107.

8. A.D.O. Bawagan, S.J. Hamrock, M. Schaberg, I. Yousef, E. Ritter, U. Schade. "Far-infrared studies on Nafion and perfluoroimide acid (PFIA) and their alkali salts". Vib. Spectrosc. 2014. 1-5. 10.1016/j.vibspec.2014.05.010.

9. M. Phonyiem, S. Chaiwongwattana, C. Lao-ngam, K. Sagarik. "Proton transfer reactions and dynamics of sulfonic acid group in Nafion ${ }^{\circledR}$." Phys. Chem. Chem. Phys. 2011. 13(23): 1092310939. 10.1039/c1cp20469f.

10. D. Horinek, S.I. Mamatkulov, R.R. Netz. "Rational design of ion force fields based on thermodynamic solvation properties”. J. Chem. Phys. 2009. 130(12): 124507-1-124507-21. 10.1063/1.3081142.

11. J.A. Elliott, S.J. Paddison. "Modelling of morphology and proton transport in PFSA membranes." Phys. Chem. Chem. Phys. 2007. 9(21): 2602-2618. 10.1039/b701234a.

12. T.H. Yu, Y. Sha, W.-G. Liu, B. V. Merinov, P. Shirvanian, W.A. Goddard. "Mechanism for degradation of Nafion in PEM fuel cells from quantum mechanics calculations." J. Am. Chem. Soc. 2011. 133(49): 19857-19863. 10.1021/ja2074642.

13. M.K. Gunde. "Optical Effects in IR Spectroscopy: Thickness-Dependent Positions of Absorbance Lines in Spectra of Thin Films". Appl. Spectrosc. 1992. 46(2): 365-372. 10.1366/0003702924125429.

14. T.G. Mayerhöfer, H. Mutschke, J. Popp. "The Electric Field Standing Wave Effect in Infrared Transmission Spectroscopy". Chem. Phys. Chem. 2017. 18(20): 2916-2923. 10.1002/cphc.201700688. 
15. A. Kučírková, K. Navrátil. "Undesirable Occurrence of the Interference Fringes in IR Transmittance Spectra”. Scr. Fac. Sci. Nat. Univ. Masaryk. Brun. 1993. 23: 5-27.

16. B.D. Teolis, M.J. Loeffler, U. Raut, M. Famá, R.A. Baragiola. "Infrared reflectance spectroscopy on thin films: Interference effects". Icarus. 2007. 190(1): 274-279. 10.1016/j.icarus.2007.03.023.

17. F. Neri, G. Saitta, S. Chiofalo. "A simple procedure to remove the interference fringes from optical spectra”. J. Phys. E. 1987. 20(7): 894-896. 10.1088/0022-3735/20/7/015.

18. P.J. Farrington, D.J.T. Hill, J.H. O'Donnell, P.J. Pomery. "Suppression of Interference Fringes in the Infrared Spectra of Thin Polymer Films". Appl. Spectrosc. 1990. 44(5): 901-903. 10.1366/0003702904087163.

19. C.J. Gamsky, G.R. Howes, J.W. Taylor. "Infrared Reflection Absorption Spectroscopy of Photoresist Films on Silicon Wafers: Measuring Film Thickness and Removing Interference Fringes". Anal. Chem. 1994. 66(7): 1015-1020. 10.1021/ac00079a015.

20. R. Brendel. "The concept of effective film thickness for the determination of bond concentrations from IR spectra of weakly absorbing thin films on silicon". J. Appl. Phys. 1991. 69(11): 7395-7399. 10.1063/1.347552.

21. C.D. Mansfield, H.N. Rutt. "Evaluation of multiple beam interference effects in infrared gas spectroscopy". Meas. Sci. Technol. 1999. 10(3): 206-210. 10.1088/0957-0233/10/3/016.

22. M.E. Ziaei, B.P. Clayman. "Wedge-shaped polyethylene far-infrared windows". Appl. Opt. 1992. 31(4): 437-438. 10.1364/AO.31.000437.

23. R.F. Edgar, B.J. Stay. Techniques for suppressing optical interference errors in infrared film thickness gauging. Proc. SPIE 0590, Infrared Technology and Applications. 1985. Pp. 316-321. 10.1117/12.952000.

24. V.T. Teng, W.H. Shumaker. "A Technique for Eliminating Interference Fringes from Infrared Spectra of Thin Polymer Films". Appl. Spectrosc. 1986. 40(3): 421-422. 10.1366/0003702864509213.

25. C. Lutinski. "Method for Elimination of Interference Fringes in Spectra of Thin Films". Anal. Chem. 1958. 30(12): 2071-2072. 10.1021/ac60144a650.

26. P. Dore, A. Nucara, D. Cannavò, G. De Marzi, P. Calvani, A. Marcelli, R.S. Sussman, A.J. Whitehead, C.N. Dodge, A.J. Krehan. "Infrared properties of chemical-vapor deposition polycrystalline diamond windows". Appl. Opt. 1998. 37(24): 5731. 10.1364/AO.37.005731.

27. N.J. Harrick. "Transmission Spectra without Interference Fringes". Appl. Spectrosc. 1977. 31(6): 548-549. 10.1366/000370277774464165.

28. P. Bassan, J. Lee, A. Sachdeva, J. Pissardini, K.M. Dorling, J.S. Fletcher, A. Henderson, P. Gardner. "The inherent problem of transflection-mode infrared spectroscopic microscopy and the ramifications for biomedical single point and imaging applications". Analyst. 2013. 138(1): 144-157. 10.1039/C2AN36090J.

29. T.A. Zawodzinski, M. Neeman, L.O. Sillerud, S. Gottesfeld. "Determination of water diffusion coefficients in perfluorosulfonate ionomeric membranes". J. Phys. Chem. 1991. 95(15): 60406044. 10.1021/j100168a060.

30. M. Abo-Bakr, J. Feikes, K. Holldack, P. Kuske, W. Peatman, U. Schade, G. Wüstefeld, H.-W. Hübers. "Brilliant, Coherent Far-Infrared (THz) Synchrotron Radiation". Phys. Rev. Lett. 2003. 90(9): 094801-1-094801-4. 10.1103/PhysRevLett.90.094801.

31. K. Schuster. "Anwendung der Vierpoltheorie auf die Probleme der optischen Reflexionsminderung, Reflexionsverstärkung und der Interferenzfilter." Ann. Phys. 1949. 439(6): 352-356. 10.1002/andp.19494390607. 
32. F. Abelès. "Recherches sur la propagation des ondes électromagnétiques sinusoïdales dans les milieux stratifiés". Ann. Phys. (Paris). 1950. 12(5): 596-640. 10.1051/anphys/195012050596.

33. H. Wolter. "Optik dünner Schichten". In: S. Flügge, editor. Grundlagen der Optik. Berlin: Springer-Verlag, 1956. Pp. 461-554.

34. T.G. Mayerhöfer, H. Mutschke, J. Popp. "Employing Theories Far beyond Their Limits-The Case of the (Boguer-) Beer-Lambert Law". Chem. Phys. Chem. 2016. 1948-1955.

10.1002/cphc.201600114.

35. J.R. Birch. "The far-infrared optical constants of polypropylene, PTFE and polystyrene". Infrared Phys. 1992. 33(1): 33-38. 10.1016/0020-0891(92)90052-U.

36. K.W. Johnson, J.F. Rabolt. "Far infrared measurements on the $19^{\circ} \mathrm{C}$ phase transition in polytetrafluoroethylene". J. Chem. Phys. 1973. 58(10): 4536-4538. 10.1063/1.1679016.

37. C.Y. Liang, S. Krimm. "Infrared Spectra of High Polymers. III. Polytetrafluoroethylene and Polychlorotrifluoroethylene". J. Chem. Phys. 1956. 25(3): 563. 10.1063/1.1742964.

38. J. Rabolt, B. Fanconi. "Raman scattering from finite polytetrafluoroethylene chains and a highly oriented TFE-HFP copolymer monofilament". Macromolecules. 1978. 11(4): 740-745. 10.1021/ma60064a025.

39. A. Röseler. Infrared Spectroscopic Ellipsometry. Berlin: Akademie-Verlag Berlin, 1990. P. 15.

40. M.A. Ordal, L.L. Long, R.J. Bell, S.E. Bell, R.R. Bell, R.W. Alexander, C.A. Ward. “Optical properties of the metals $\mathrm{Al}, \mathrm{Co}, \mathrm{Cu}, \mathrm{Au}, \mathrm{Fe}, \mathrm{Pb}, \mathrm{Ni}, \mathrm{Pd}, \mathrm{Pt}, \mathrm{Ag}, \mathrm{Ti}$, and $\mathrm{W}$ in the infrared and far infrared". Appl. Opt. 1983. 22(7): 1099-1120. 10.1364/AO.22.001099.

41. P.D. Cunningham, N.N. Valdes, F.A. Vallejo, L.M. Hayden, B. Polishak, X.H. Zhou, J. Lou, A.K.-Y. Jen, J.C. Williams, R.J. Twieg. "Broadband terahertz characterization of the refractive index and absorption of some important polymeric and organic electro-optic materials". J. Appl. Phys. 2011. 109(4): 1-5. 10.1063/1.3549120.

42. I.S. Gradstein, I.M. Ryshik. Tablizy Integralow, Summ, Rjadow i Proiswedenij. Moskwa: Gos. Isdat. Fyz.-Mat. Lit., 1963. P. 54.

\section{Figure Captions:}

Figure 1: The calculated absorbance spectra of a non-absorbing polymer film with a nominal thickness of $50 \mu \mathrm{m}$ and a refractive index of 1.4 for the far and mid infrared regions. The dotted curve is obtained for parallel film boundaries while the solid curve is obtained for a wedged film with varying thicknesses from 45 to $55 \mu \mathrm{m}$ applying equation A6.

Figure 2: Scheme for the TTR measurement, where the normal of the incoming plane wave, $E_{0}(v)$, has an angle of incidence of $\varphi_{0}$ to the film normal and is back reflected as a planar wave, $E_{r}(v)$. The film has a complex refractive index of $\tilde{n}$ and a thickness of $x$ and is placed at a distance $d$ in front of a plane mirror. The refractive index of the ambient is considered to be unity and the mirror reflectivity over the frequency range of concern is $100 \%$.

Figure 3: Transmittance absorbance spectra (left) and TTR absorbance spectra (right) of PTFE films of different thicknesses. Note, that the apparent band at around $203 \mathrm{~cm}^{-1}$ in the TTR absorbance spectra moves towards higher energies and starts to saturate with increasing film thickness.

Figure 4: Calculated absorption spectra for transmittance (left) and TTR (right) geometry using a film model and a harmonic oscillator at $200 \mathrm{~cm}^{-1}$ as described in the text. 
Figure 5: Absorbance maximum of the $203-\mathrm{cm}^{-1}$ and $277-\mathrm{cm}^{-1}$ band derived from the experimental PTFE spectra in Fig. 3 for both single transmittance and TTR measurement geometry. The dashed lines indicate the maximum absorbance as one expects from the Lambert-Beer's law. Note that for the TTR measurements the radiation passes the film twice and therefore yields higher absorbance values than obtained for the simple transmittance absorbance.

Figure 6: TTR absorbance spectra of Nafion NR211/ $\mathrm{K}^{+}$for a varying gap between the film and the back-mirror. The spectra are shifted for clarity.

Figure 7: Transmittance absorbance spectra and TTR absorbance spectra of Nafion NR211/K $\mathrm{K}^{+}$and the corresponding difference spectra taking into account that the radiation passes twice through the film for the TTR measurement. For comparison the calculated transmittance absorbance spectra of a nonabsorbing film of equivalent thickness is displayed.

Figure 8: Transmittance absorbance spectra and TTR absorbance spectra of Nafion NR212/Cs ${ }^{+}$and the corresponding difference spectra in comparison to the absorbance spectrum of a model film as already explained for Fig. 4.

Table 1: Absorption band position of the $\mathrm{K}^{+}$and $\mathrm{Cs}^{+}$vibrations in Nafion.

\begin{tabular}{ccccc}
\hline Cation & $\begin{array}{c}v\left(\mathrm{~cm}^{-1}\right), \\
(\mathrm{amu})\end{array}$ & $\begin{array}{c}v\left(\mathrm{~cm}^{-1}\right), \\
\text { transmittance } \\
\text { spectra, fringes } \\
\text { subtracted }\end{array}$ & $\begin{array}{c}v\left(\mathrm{~cm}^{-1}\right), \\
\text { transmittance spectra }\end{array}$ \\
\hline $\mathrm{K}^{+}$ & 39.1 & 150 & 146 & 150 \\
$\mathrm{Cs}^{+}$ & 132.9 & 83 & 93 & 90 \\
\hline
\end{tabular}


$\mathrm{THz}$

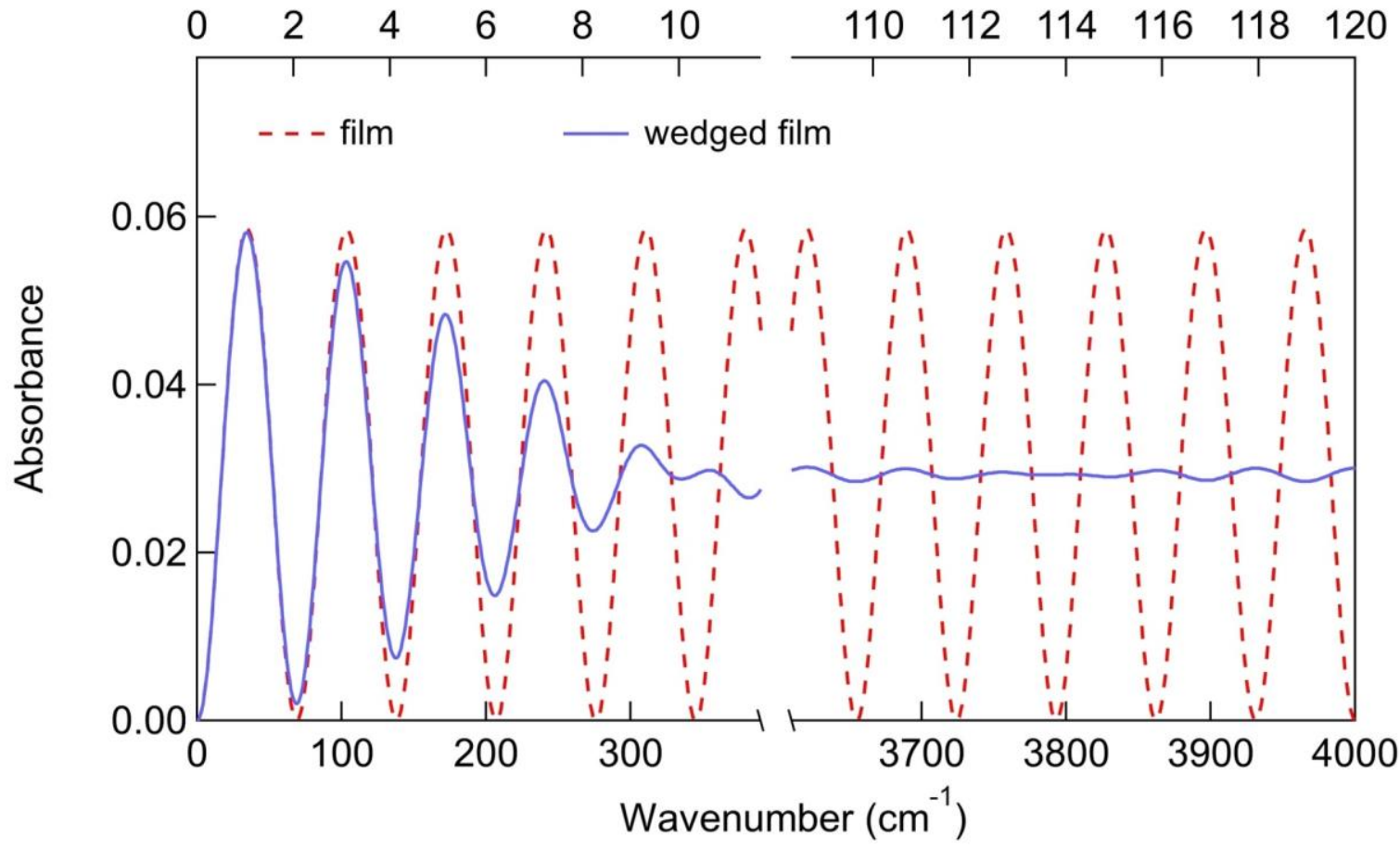

Fig 1

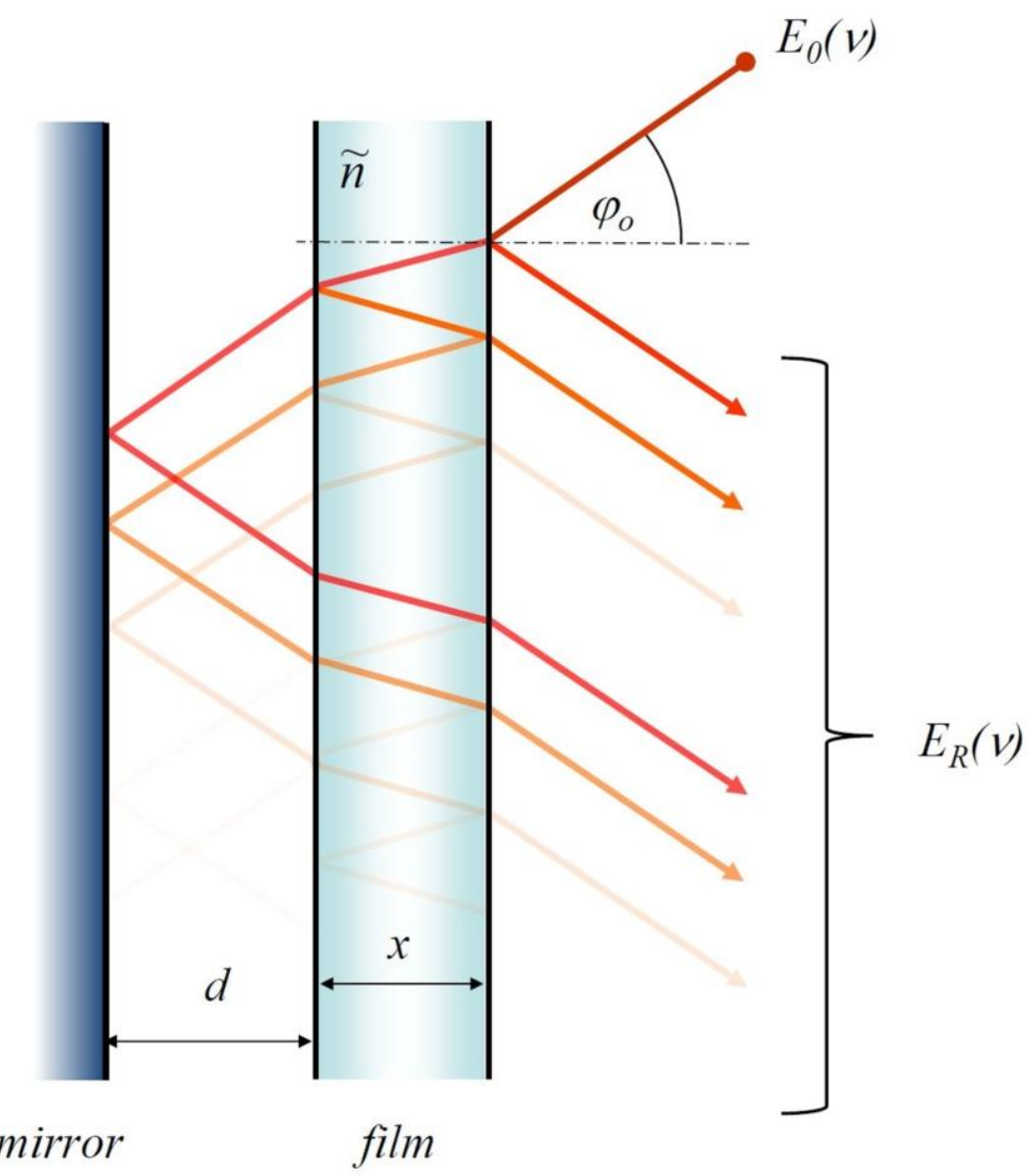

Fig. 2 

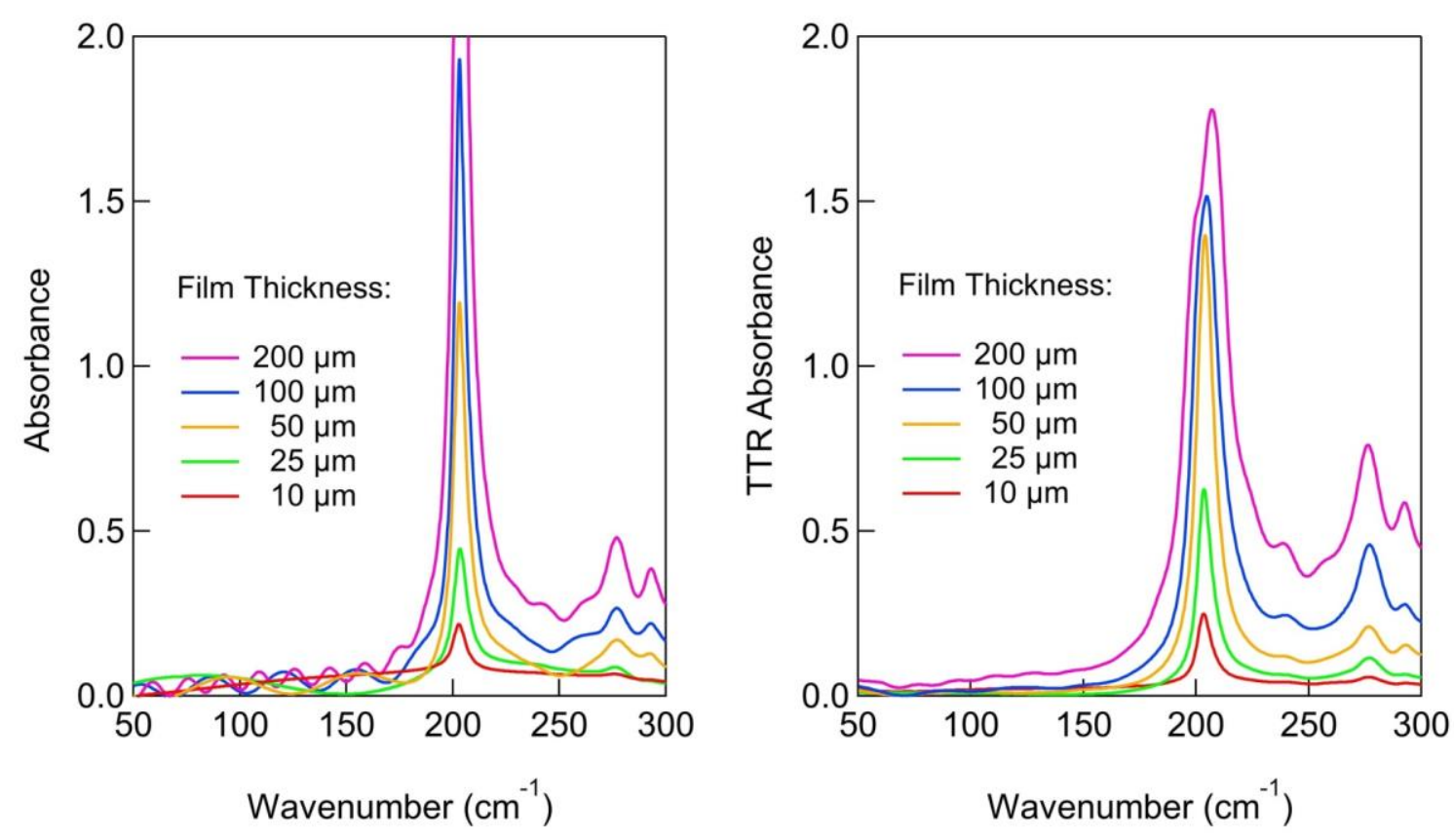

Fig. 3
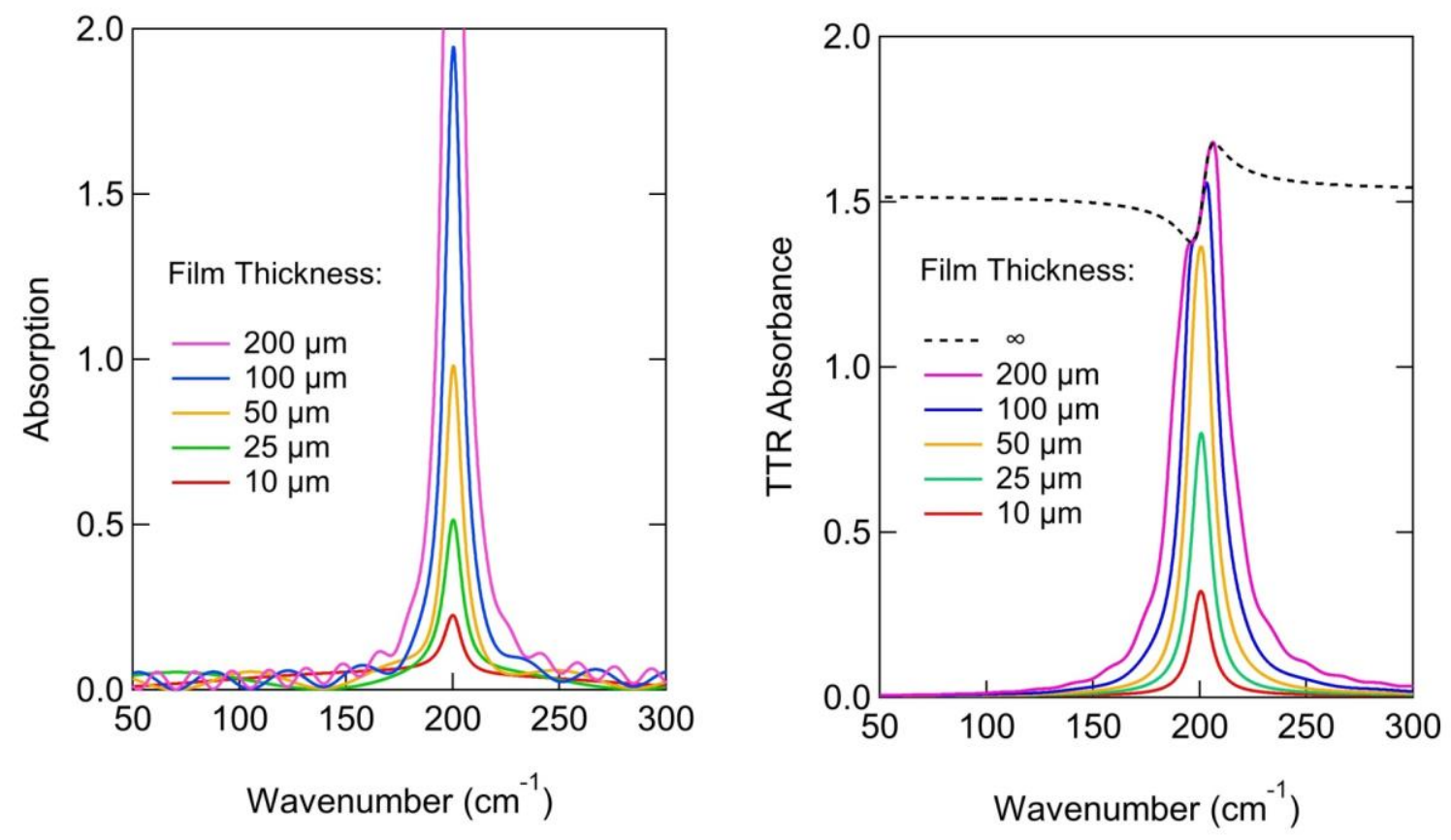

Fig. 4 

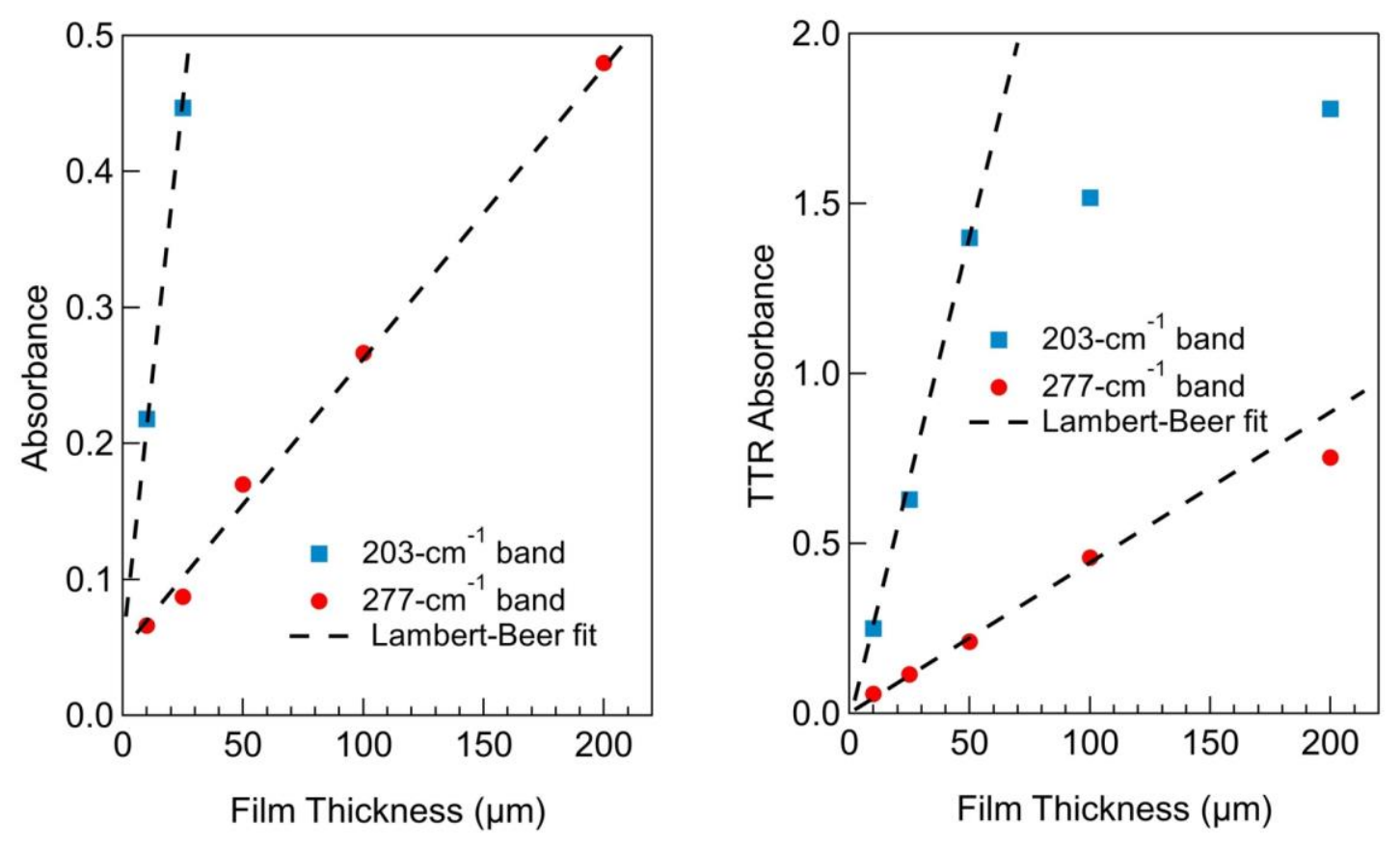

Fig. 5

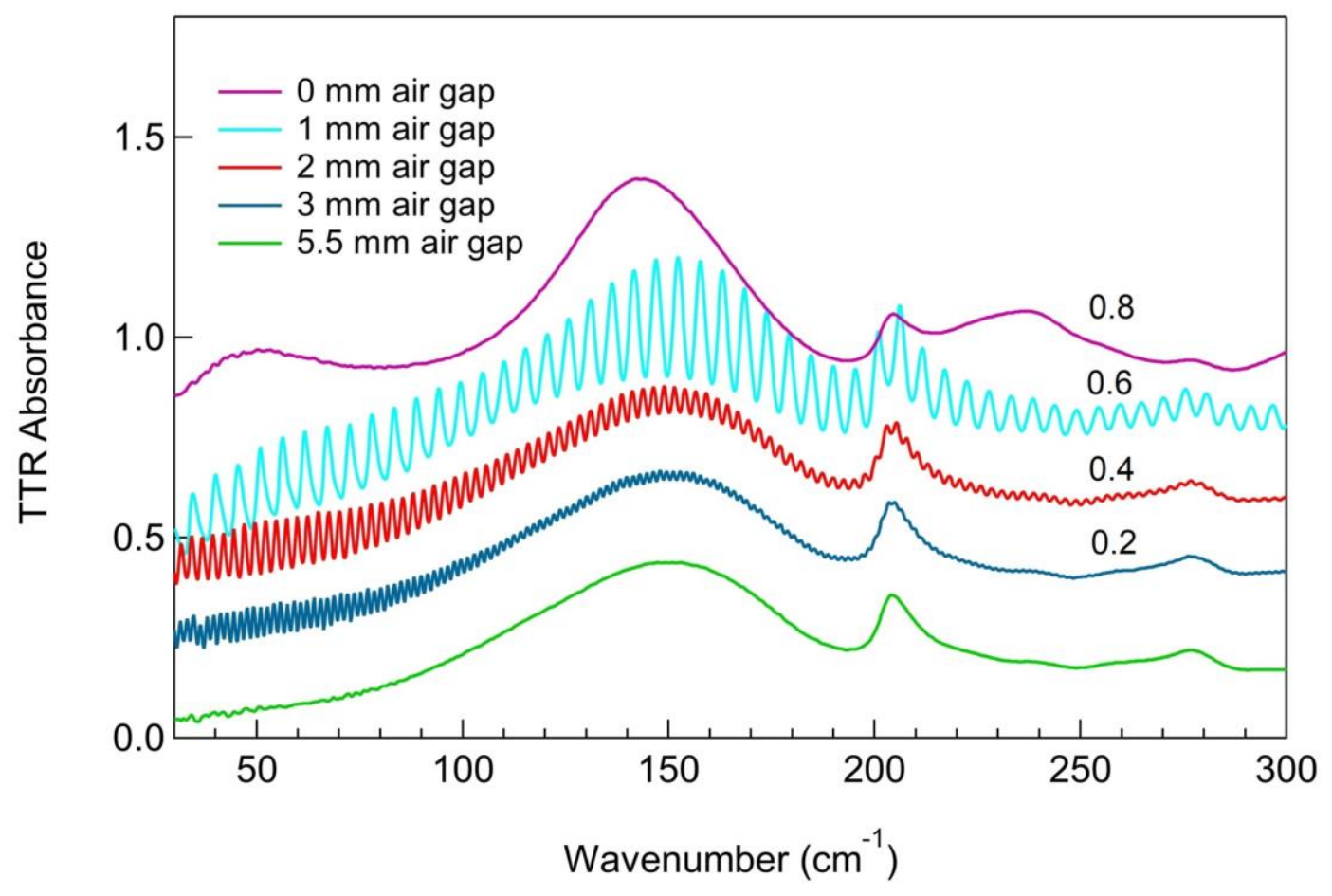

Fig. 6 


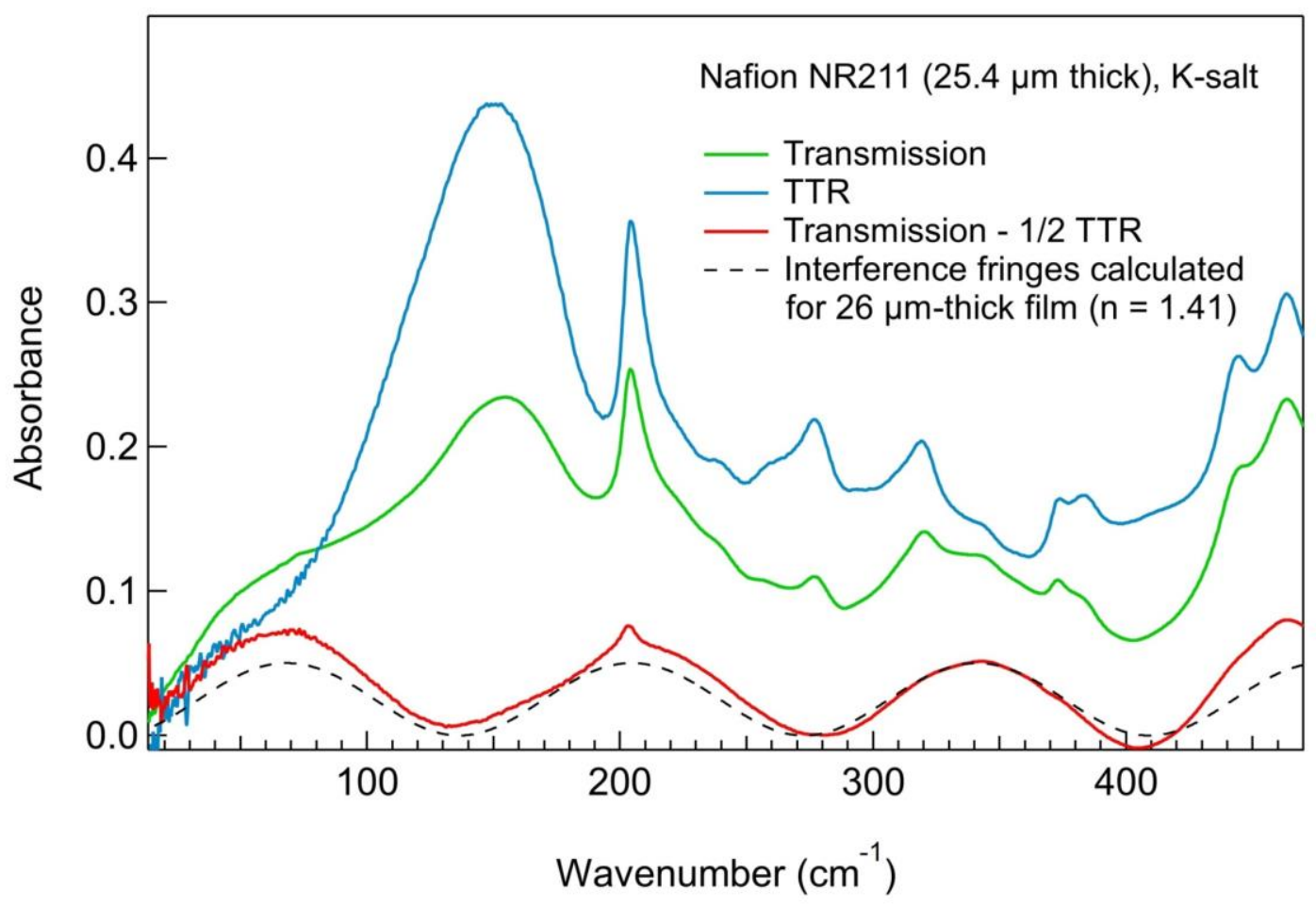

Fig. 7

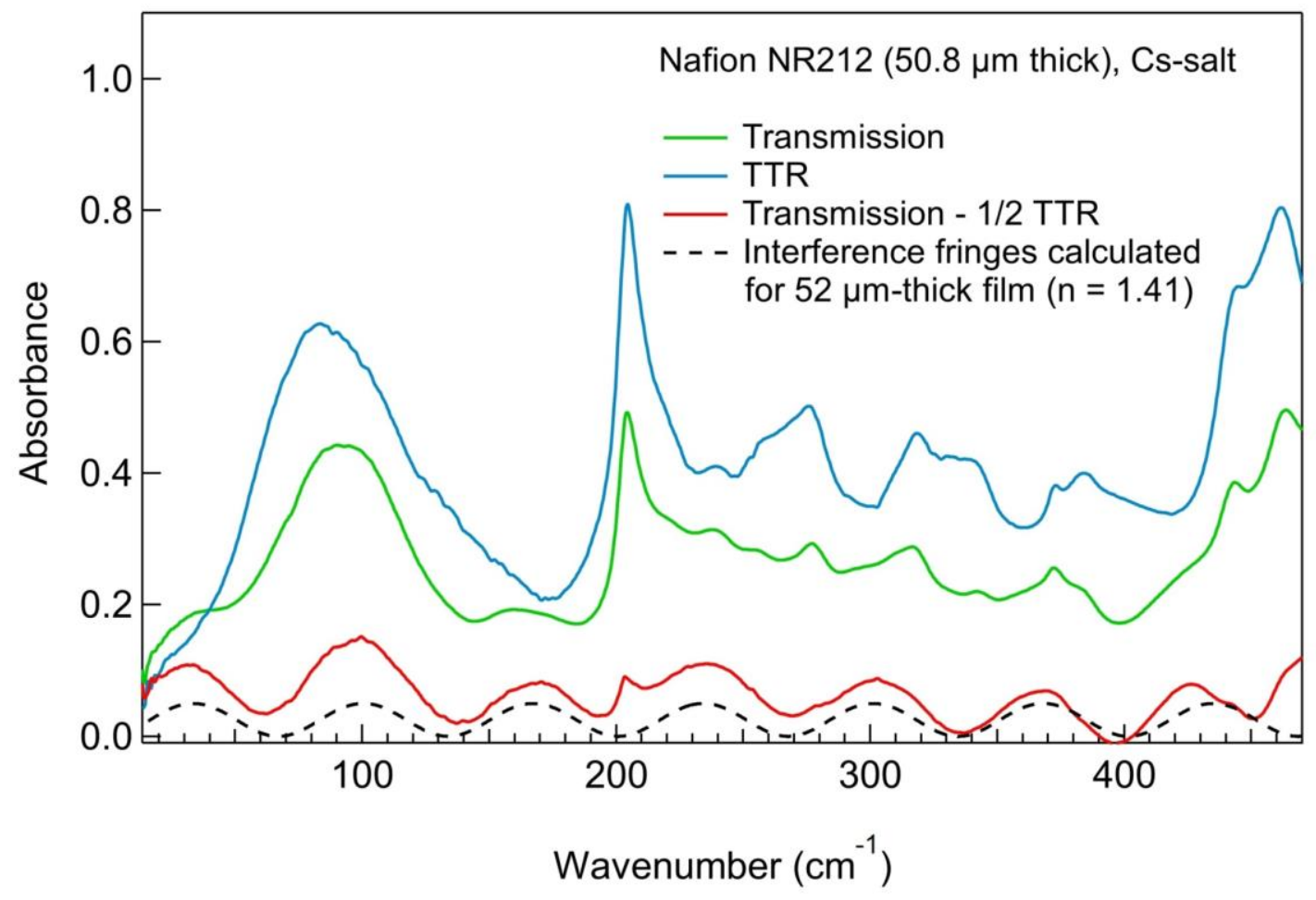

Fig. 8 